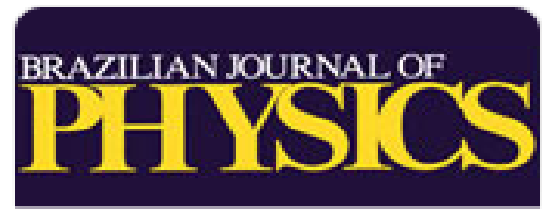

Brazilian Journal of Physics

ISSN: 0103-9733

luizno.bjp@gmail.com

Sociedade Brasileira de Física

Brasil

De Los Santos Valladares, L.; Bustamante Dominguez, A.; Albino Aguiar, J.; Reeve, R. M.; Mitrelias,

T.; Langford, R. M.; Azuma, Y.; W. Barnes, C. H.; Majima, Y.

Reorientation Response of Magnetic Microspheres Attached to Gold Electrodes Under an Applied

Magnetic Field

Brazilian Journal of Physics, vol. 43, núm. 4, agosto, 2013, pp. 209-213

Sociedade Brasileira de Física

Sâo Paulo, Brasil

Available in: http://www.redalyc.org/articulo.oa?id=46427890001

How to cite

Complete issue

More information about this article

Journal's homepage in redalyc.org

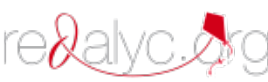

Scientific Information System

Network of Scientific Journals from Latin America, the Caribbean, Spain and Portugal

Non-profit academic project, developed under the open access initiative 


\title{
Reorientation Response of Magnetic Microspheres Attached to Gold Electrodes Under an Applied Magnetic Field
}

\author{
L. De Los Santos Valladares • A. Bustamante Dominguez • \\ J. Albino Aguiar • R. M. Reeve - T. Mitrelias - R. M. Langford • \\ Y. Azuma • C. H. W. Barnes • Y. Majima
}

Received: 22 January 2012 / Published online: 24 May 2013

(C) Sociedade Brasileira de Física 2013

\begin{abstract}
In this work, we report the mechanical reorientation of thiolated ferromagnetic microspheres bridging a pair of gold electrodes under an external magnetic field. When an external magnetic field $(7 \mathrm{kG})$ is applied during the measurement of the current-voltage characteristics of a carboxyl ferromagnetic microsphere $(4 \mu \mathrm{m}$ diameter $)$ attached to two gold electrodes by self-assembled monolayers (SAMs) of octane dithiol $\left(\mathrm{C}_{8} \mathrm{H}_{18} \mathrm{~S}_{2}\right)$, the current signal is distorted. Rather than due to magnetoresistance, this effect is caused by a mechanical reorientation of the ferromagnetic sphere, which alters the number of SAMs between the
\end{abstract}

L. De Los Santos Valladares $(\bowtie) \cdot R$. M. Reeve · T. Mitrelias •

R. M. Langford · C. H. W. Barnes

Cavendish Laboratory, Department of Physics,

University of Cambridge, J.J. Thomson Av.,

Cambridge CB3 0HE, UK

e-mail: luis_d_v@hotmail.com

L. Valladares

e-mail: luisitodv@yahoo.es

L. De Los Santos Valladares · Y. Azuma · Y. Majima

Materials and Structures Laboratory,

Tokyo Institute of Technology, 4259 Nagatsuta-cho, Midori-ku,

Yokohama 226-8503, Japan

L. De Los Santos Valladares · J. A. Aguiar

Departamento de Física, Universidade Federal de Pernambuco, 50670-901, Recife-Pe, Brazil

A. B. Dominguez

Laboratorio de Cerámicos y Nanomateriales, Facultad de Ciencias

Físicas, Universidad Nacional Mayor de San Marcos,

Ap. Postal 14-0149, Lima, Peru

Y. Majima

CREST, Japan Science and Technology Agency (JST),

4259 Nagatsuta-cho, Midori-ku,

Yokohama 226-8503, Japan sphere and the electrodes and therefore affects conduction. To study the physical reorientation of the ferromagnetic particles, we measure their hysteresis loops while suspended in a liquid solution.

Keywords Magnetic microparticles (75.50.Tt) Electric current measurement $(84.37 .+q) \cdot$ Magnetic hysteresis (75.60.-d)

\section{Introduction}

As part of the continued drive to shrink electronics circuits and preserve Moore's law [1], researchers have been able to attach individual molecules and nanostructures to a pair of nanogap electrodes to form single-electron transistors (SETs) and measured the resulting electrical conductance. In SETs, the conductance depends on the asymmetry between the couplings to the two electrodes, instead of on the average coupling [2]. Unfortunately, the fabrication of reliable SETs poses enormous challenges. Even if great care is taken during assembly, one cannot be sure that a particular molecule has been positioned at the junction, because direct observation is impossible. Experimental results are often distinct from theoretical predictions, and accurate descriptions of the characteristics of particular structures are difficult to offer. For instance, recently, molecular switching was observed in endohedral metallofullerene which, following careful analysis of the current-voltage characteristics, was traced to a reorientation of the molecule driven by the interaction between its electric dipole moment and an external electric field [3]. Even more challenging are single molecules or nanostructures carrying magnetic moments, so that only a few experiments have succeeded in fabricating SETs based on magnetic structures [4-6]. 
By contrast, it is easier to assemble microstructures on metal surfaces $[7,8]$. Studies of these reliable systems, with easier-to-interpret characteristics, are expected to offer prior understanding of more complex systems, such as SETs. Here, we report the reorientation of a ferromagnetic microsphere attached to a pair of gold electrodes and its effect on current-voltage characteristics under an external magnetic field. We work with microscale dimensions, which dwarf the typical-less than $5 \mathrm{~nm}[3-6,9]$-SET size. Our device is therefore better described by classical models than by quantum mechanical ones.

\section{Experimental}

The fabrication of the samples comprised a number of steps. Photolithographically defined arrays of gold electrode pairs, separated by $2 \mu \mathrm{m}$ gaps, were patterned by a conventional evaporation and lift-off process. Small Au pellets (99.999 \% purity) were evaporated from a tungsten boat on polished $\mathrm{SiO}_{2}(80 \mathrm{~nm}) / \mathrm{Si}(100)$ substrates using an Edwards 306 evaporator system with a base pressure of $10^{-7}$ mbar. The rate of evaporation was maintained at $0.1 \mathrm{~nm} \mathrm{~s}^{-1}$ and the thickness of gold (40 nm) was controlled by a quartz crystal microbalance located next to the substrate. The gold electrodes were functionalized with $\mathrm{C}_{8} \mathrm{H}_{18} \mathrm{~S}_{2}$ (octane dithiol, Alfa Aesar B24693) following standard procedures [10]. In parallel, carboxyl ferromagnetic spheres of mean diameter $4 \mu \mathrm{m}$ (CFM-40-10, Spherotech Inc.) with polystyrene cores and iron oxide shells were dried, suspended in distilled water or coated with octane dithiol $\left(\mathrm{C}_{8} \mathrm{H}_{18} \mathrm{~S}_{2}\right)$. The latter were obtained by dispersing and mixing the ferromagnetic spheres in $0.5 \mathrm{~mL}$ of $2 \mathrm{mM}$ octane dithiol in ethanol for $48 \mathrm{~h}$. During this process, some spheres stuck together forming groups, and we observed that the number of groups increases with time and molarity. The coated spheres were transferred to a beaker containing $5 \mathrm{~mL}$ of ethanol and the functionalized electrodes. The system was left for $48 \mathrm{~h}$ at room temperature (RT) and finally rinsed with large amounts of ethanol to remove the unlinked spheres from the substrate. The final sample consisted of thiolated ferromagnetic microspheres which are physisorbed on functionalized gold electrodes and creating bridges. Current-voltage (I-V) measurements were made in a probe station Grail10-205-LV Nagase Techno-Engineering Co. LTD, at RT and in a high vacuum, of $8.6 \times 10^{-3} \mathrm{~Pa}$. Two $\mathrm{BeCu}$ electrodes (source (S) and drain (D)) were in direct contact with a pair of the gold electrodes, and the current response was sensed in the drain electrode after applying a source-drain bias in the range of -5 to $+5 \mathrm{~V}$, in forward and reverse modes. The magnet setup allowed us to apply an in-plane $7 \mathrm{kG}$ external field, parallel to the current. Due to lack of refrigerant (helium), we were unable to study the thermal dependence of the current-voltage characteristics.

In order to understand the mechanical motion of the ferromagnetic spheres under external magnetic fields, we suspended the samples in liquid solution and measured the magnetic hysteresis loops in a Magnetic Property Measurement System (MPMS-SQUID) from Quantum Design. Since the density of the distilled water is too low to hold the spheres in suspension over the long periods, 4$6 \mathrm{~h}$, required to acquire data from the magnetometer, the spheres were suspended in a sucrose liquid solution as described in our previous work [11]. In brief, $3 \mathrm{~g}$ of sucrose (sigma Ultra 99.55 GC) were diluted in $6 \mathrm{~mL}$ of distilled water to suspend the spheres and to avoid sedimentation. Since the standard holder for the MPMS, a gelatine capsule, is designed to sustain powder and dried samples, we had to fabricate a special sample holder, a diamagnetic cone plastic, to hold the liquid solution [11]. A drop of approximately $1 \mu \mathrm{L}$ of spheres suspended in the solution was measured in the MPMS equipment.

\section{Results and Discussions}

Figure 1 shows arrays of functionalized gold electrodes with CFM microspheres physisorbed through SAMs of octane dithiol. The linking of the thiols to the gold occurs by a
Fig. 1 Optical microscope images of multiple microspheres (magnification $\times 50($ a) $)$ and individual microspheres of $4 \mu \mathrm{m}$ mean diameter (magnification $\times 75$ (b)) bridging gold electrodes
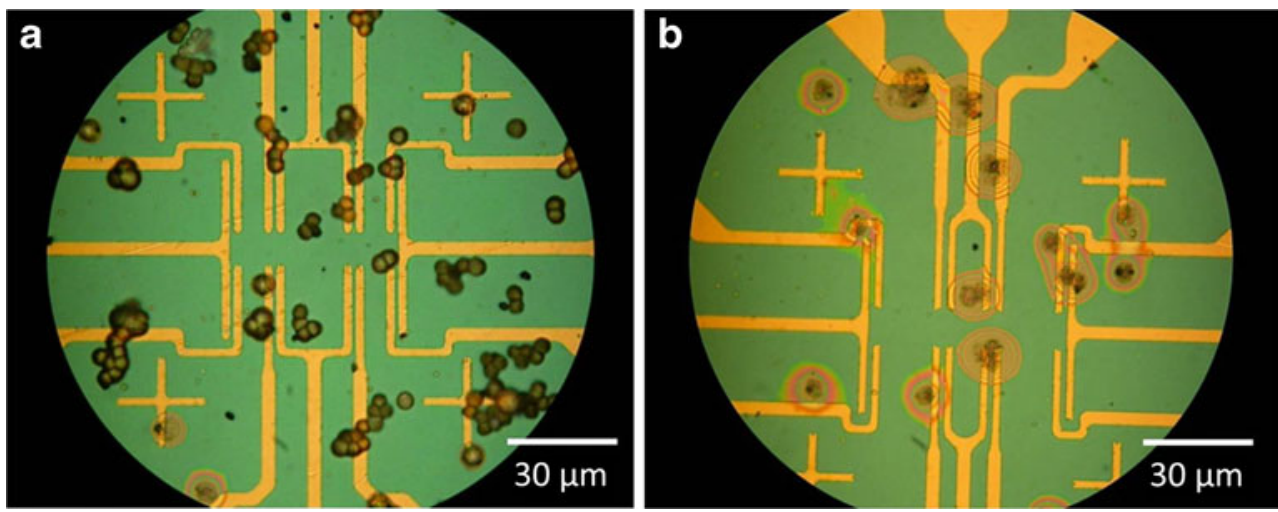
combination of dative bonding (chemisorption) between sulfur and gold, as it has been reported elsewhere [10]. The spheres are immobilized relative to the electrodes by the strong affinity of the sulfur atoms of the functional group to gold, whereas intermolecular van der Waals forces are responsible for the lateral packing of the layers [10]. The strong adhesion of the spheres to the electrodes was tested via several washings of ethanol, propanol, and distilled water. Our fabrication technique therefore insures that the spheres do not detach from the electrodes when the external magnetic field is applied. In Fig 1a, the optical microscope image at $\times 50$ magnification shows groups of thiolated microspheres indicating long-time functionalization. In contrast, in Fig. $1 \mathrm{~b}$, individual microspheres are attached to the gold electrodes. This was achieved by adjusting the functionalization time to $48 \mathrm{~h}$. The less distinct image of the microspheres in Fig $1 \mathrm{~b}$ is caused by the diffraction of light through the optical microscope at the chosen magnification $(\times 75)$.

Figure 2 shows the $\mathrm{I}-\mathrm{V}$ response of an arbitrary individual iron-coated polystyrene (CFM) sphere attached to two gold electrodes. The measurements were performed from -5 to $+5 \mathrm{~V}$ in forward and reversed bias as indicated by the arrows inset in the figure. Initially, in the absence of the sphere, a tunneling current is detected, and the forward and reverse bias curves overlap, as shown in Fig. 2a. The persistence of this superposition after several scans indicates that the system is mechanically and electrically stable. After the electrodes are bridged with a thiolated microsphere, a similar tunneling current, forward and reverse bias currents that also overlap, is observed, but the magnitude of the current is amplified. The amplification is due to by the higher conductivity of the SAMs-sphere-SAMs element bridging the source and drain electrodes, which lowers the

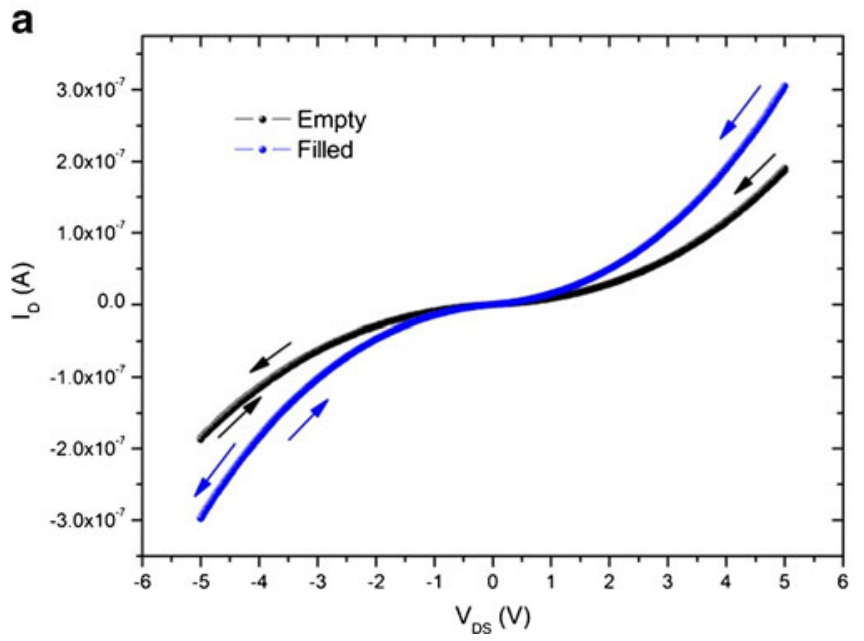

Fig 2 I-V responses of an arbitrary CFM microsphere bridging two gold electrodes through octane dithiol SAMS. a Comparison between the I-V signals before (black) and after (blue) bridging the electrodes electrical resistance of the device in comparison with the empty case, as one would expect.

Application of the external magnetic field changes the picture. As shown in Fig. 2b, as soon an external magnetic field of $7 \mathrm{kG}$ is switched on, the current-voltage curves are distorted. The forward bias curve no longer matches the reverse bias plot, except near-zero bias. In fact, our measurements show that the zero bias conductance is null, an indication that no leakage current flows through the system and that the effect comes from the device, not to from the equipment used in the $\mathrm{I}-\mathrm{V}$ characterization. Changes in the resistance under magnetic fields, i.e., magneto resistance, have widely been observed, especially in direct charge transport through iron SETs [12, 13]. As mentioned above, however, the device described in this work is not a SET. Instead, the bridging element is a $4 \mu \mathrm{m}$ polystyrene (core) iron oxide (shell) sphere attached to the goldelectrodes by multiple SAMs of octane dithiol. The transport mechanism in the microspheres is very different from conduction in SETs. Moreover, it is uncertain whether the current penetrates the carboxyl/iron coating to the polystyrene core; the current may well flow through the SAMs and carboxyl surface, instead. The distortion in the I-V characteristics of the device cannot therefore be attributed to magneto resistance. One alternative is magnetic-field induced mechanical reorientation of the microsphere, which is expected to reduce the number of SAMs between the electrodes and to distort the $\mathrm{I}-\mathrm{V}$ characteristics.

To better understand the mechanical movement of the ferromagnetic microspheres under the external field, magnetic-hysteresis loops of immobilized and suspended microspheres were studied. Immobilized hysteresis loops were obtained from dried and packed microspheres, whereas

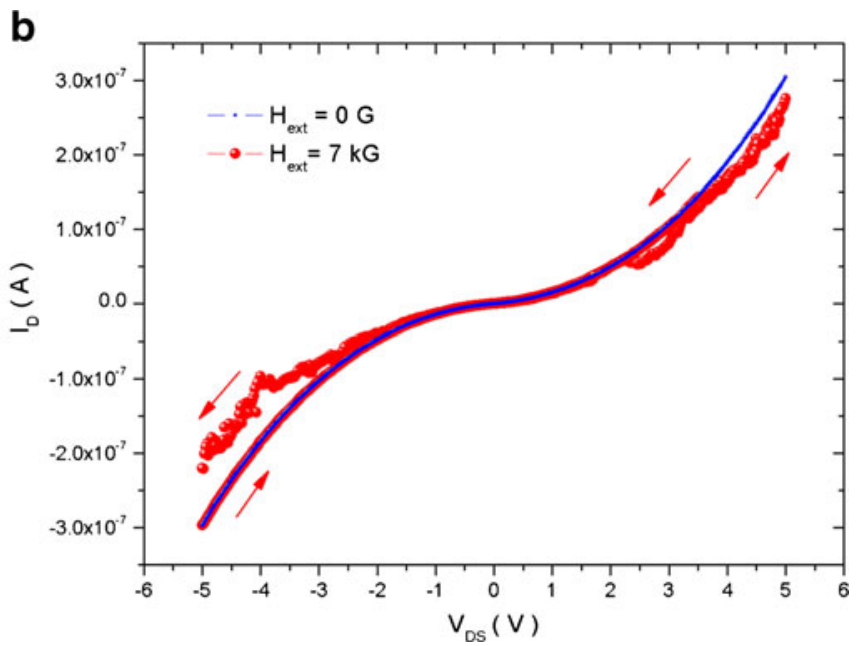

with a microsphere. b Comparison between the I-V signals through the bridged electrodes with and without an external magnetic field 
suspended hysteresis were taken after suspending them in a sucrose liquid solution, as explained before. Figure 3 shows the hysteresis loops of the magnetic microspheres under three distinct conditions: immobilized, in liquid suspension without thiol coating, and in liquid suspension after thiol coating. Figure 3a compares the hysteresis loop of the immobilized microspheres and of the suspended spheres up to $16 \mathrm{kG}$. For immobilized spheres, the plot shows the classic hysteresis loop of a ferromagnetic material. The remnant magnetization point is half of the saturation, reached around $4 \mathrm{kG}$. The scattering in the suspended-sphere loop is noise due to the motion of the spheres. For growing external field, when saturation is first reached, most of the magnetic moments of the spheres are aligned with the field; from this point on, the diamagnetism of the plastic container pushes the measured magnetization towards zero. In contrast with the loop for the immobile spheres, the hysteresis loop for the suspended spheres displays no coercive field.

Figure $3 \mathrm{~b}$ compares the hysteresis loops for immobilized ferromagnetic spheres and thiolated spheres in suspension, up to $4 \mathrm{kG}$. The behavior of the magnetization is similar to that in Fig. 3a. The hysteresis loops of the suspended ferromagnetic spheres show no coercivity because the magnetic moments abruptly switch to follow the applied magnetic field when it is reversed. Since the spheres are suspended in a liquid medium, they have more degrees of freedom (translation and rotation) than the immobile spheres. When the direction of the external magnetic field reverses, not only the magnetic moments, but the entire spheres undergo physical rotation. This process being essentially reversible, the loops exhibit zero coercivity. In the immobilized case, by contrast, physical rotation of spheres that are either packed or stuck together is impossible, and only the magnetic moments are reoriented.
As already explained, the noisy hysteresis loops signal motion of the suspended spheres. The spheres are not completely static, because the sample holder moves continuously during the data scanning by the MPMS-SQUID equipment. Therefore, the relative positions of the spheres vary. In equilibrium, the gravitational force $\mathrm{F}_{\mathrm{G}}$ on a particular sphere is balanced by the buoyancy force $\left(F_{B}\right)$. As discussed in [11], the magnetophoretical force $\left(F_{M}\right)$ generated during application of the magnetic field over the sphere is compensated by a drag force $\left(F_{D}\right)$, especially strong when the external magnetic field changes value.

An external magnetic field therefore tends to displace and rotate the CFM microspheres. Figure 4 depicts a microsphere attached to the gold electrodes. The sphere is immobilized between the gold electrodes by the octane dithiol SAMs, not shown in the figure. When the applied magnetic field is switched on, the sphere tends to move in the direction of the field. The sphere is still attached to the electrodes, however, and has translational and rotational inertia. The force due to the changing field gives rise to slight vibration, which reduces the number of SAMs in a few contact areas. The conductivity is consequently changed, as shown by Fig. 2. The response to external magnetic fields of microspheres attached to the electrodes could help the characterization of magnetic nanostructured SETs, which so far have only been studied theoretically [14].

\section{Conclusions}

In summary, while the current-voltage characteristics of single-electron devices are dictated by the modulation of molecular-bond energies, larger devices such as the one

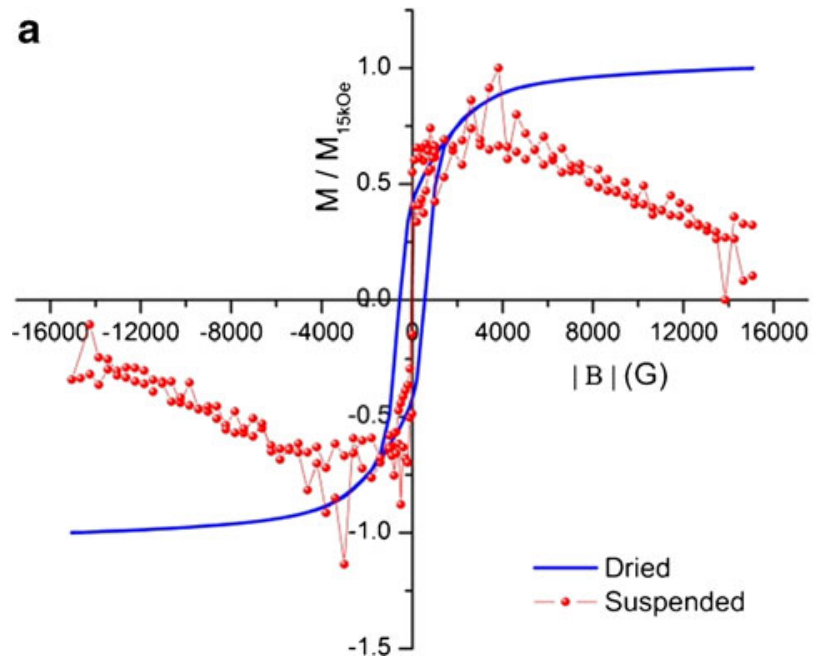

Fig. 3 Hysteresis loops of ferromagnetic microspheres under three conditions: dried, suspended, and functionalized suspended. a Comparison between dried and suspended up to $16 \mathrm{kG}$ external fields. b

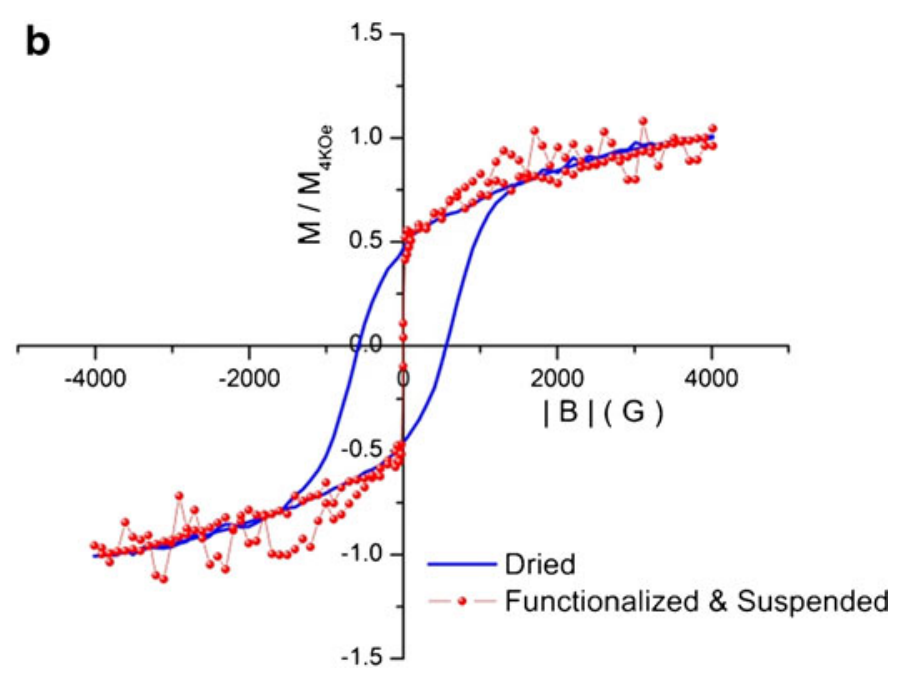

Comparison between dried and functionalized and suspended up to $4 \mathrm{kG}$ fields. In both cases, the absence of coercivity indicates that the spheres rotate under magnetic-field reversal 


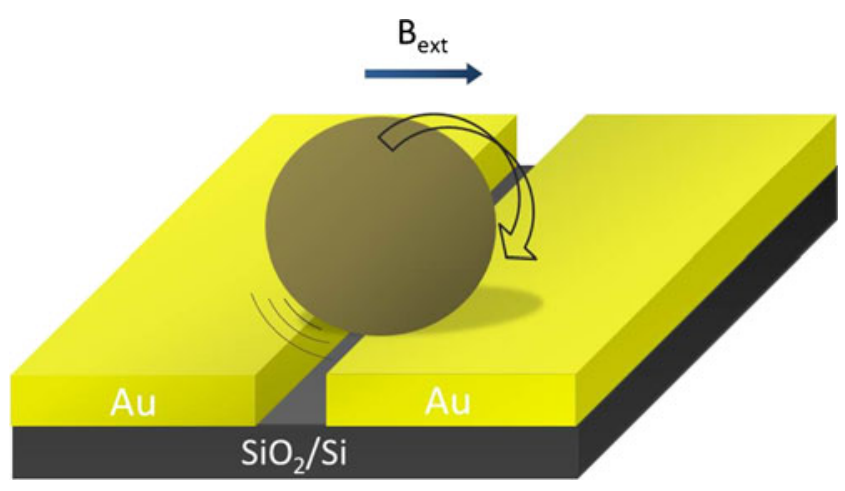

Fig 4 Reorientation of a ferromagnetic microsphere attached to two gold electrodes by self-assembled monolayers of alkane dithiol (not shown) under an external magnetic field $B_{\text {ext }}$

discussed in this work display simpler I-V curves. Quantum conductance effects are washed out by the relatively large dimensions of the components and number of links between the sphere and each electrode. The magnetic field dependence of the I-V plots in Figs. 2 on and the dependence of the mobility of the spheres in Fig. 3 have therefore been explained on the basis of the classical model depicted in Fig. 4. The vibrational motion of the microsphere in response to the application of the external magnetic field reduces the number of SAMs linking the sphere to the electrodes, and the resulting change in conductance distorts the current-voltage characteristics.

Acknowledgments This work was supported by the European Union Program ALBAN (grant no. E06D101257PE) and the Cambridge Overseas Trust. The work in Brazil was supported by CAPES. The work in Japan was supported by the Japan Society for the Promotion of Science (Furusato FU036), Grants-in-Aid for Scientific Research on Innovative Areas (no. 20108011, $\pi$-Space) from the Ministry of
Education, Culture, Sports, Science and Technology (MEXT), a Collaborative Research Project of the Materials and Structures Laboratory, Tokyo Institute of Technology and the World Class University Program, Sunchon National University, Korea. The authors are indebted to Dr. Norio Okabayashi from the Tokyo Institute of Technology and Miss Lizbet Leon from the University of Brasilia for their help during different stages of the experiment.

\section{References}

1. G.E. Moore, Electronics 38, 114 (1965)

2. L.I. Glazman, M.E. Raikh, JETP Lett. 47, 452-455 (1988)

3. Y. Yasutake, Z. Shi, T. Okazaki, H. Shinohara, Y. Majima, Nano Lett. 5, 1057-1060 (2005)

4. N. Roch, R. Vincent, F. Elste, W. Harneit, W. Wernsdorfer, C. Timm, F. Balestro, Phys. Rev. B 83, 081407 (2011)

5. A.S. Zyazin, J.W.G. van den Berg, E.A. Osorio, H.S.J. van der Zant, N.P. Konstantinidis, M. Leijnse, M.R. Wegewijs, F. May, W. Hofstetter, C. Danieli, A. Cornia, Nano Lett. 10, 3307 (2010)

6. L. Bogani, W. Wernsdorfer, Nature Mater. 7, 179-186 (2008)

7. D.P. Long, C.H. Patterson, M.H. Moore, D.S. Seferos, G.C. Bazan, J.G. Kushmerick, Appl. Phys. Lett. 86, 153105 (2005)

8. L. De Los Santos Valladares, A. Bustamante Dominguez, J. Llandro, S. Suzuki, T. Mitrelias, R. Bellido Quispe, C.H.W. Barnes, Y. Majima, Jpn. J. Appl. Phys. 49, 093102 (2010)

9. L. De Los Santos Valladares, L. Leon Felix, A. Bustamante Dominguez, T. Mitrelias, F. Sfigakis, S.I. Khondaker, C.H.W. Barnes, Y. Majima, Nanotechnology 21, 445304 (2010)

10. A. Ulman, Chem. Rev. 96, 1533-1554 (1996)

11. L. De Los Santos Valladares, J. Llandro, D. Lee, T. Mitrelias, J.J. Palfreyman, T.J. Hayward, J. Cooper, J.A.C. Bland, C.H.W. Barnes, J. Arroyo, M. Lees, J. Magn. Magn. Mat. 321, 2129 2134 (2009)

12. Z. Guo, H.T. Hahn, H. Lin, A.B. Karki, D.P. Young, J. Appl. Phys. 104, 014314 (2008)

13. S.S.P. Parkin, Annu. Rev. Mater. Sci. 25, 357-388 (1995)

14. X. Waintal, P.W. Brouwer, Phys. Rev. Lett. 91, 247201 (2003) 